\title{
Philosophiques
}

\section{La place du normatif en morale}

\section{Bernard Baertschi}

Volume 28, numéro 1, printemps 2001

La nature des normes

URI : https://id.erudit.org/iderudit/004895ar

DOI : https://doi.org/10.7202/004895ar

Aller au sommaire du numéro

Éditeur(s)

Société de philosophie du Québec

ISSN

0316-2923 (imprimé)

1492-1391 (numérique)

Découvrir la revue

\section{Citer cet article}

Baertschi, B. (2001). La place du normatif en morale. Philosophiques, 28(1), 69-86. https://doi.org/10.7202/004895ar

\section{Résumé de l'article}

On a reproché au modèle perceptuel de la connaissance morale d'être inadéquat en ce qu'il serait incapable d'expliquer le signe distinctif et fondamental de l'éthique, à savoir son caractère normatif. Je tente de montrer que la critique n'est pas pertinente, car le normatif n'a en réalité qu'une place dérivée en morale : l'éthique est d'abord une question de valeurs, entités dont il est tout à fait plausible de dire que nous les percevons. Pour justifier la place dérivée du normatif, je m'appuie sur les études de Max Scheler et sur l'examen de certains traits de la psychologie morale, qui tendent à montrer que le normatif n'est qu'un cas particulier de l'axiologique, mettant en évidence les caractères de force des valeurs, de risques de transgression et de contrainte. 


\title{
La place du normatif en morale
}

\author{
BERNARD BAERTSCHI \\ Université de Genève \\ bernard.baertschi@lettres.unige.ch
}

\begin{abstract}
RÉSUMÉ. - On a reproché au modèle perceptuel de la connaissance morale d'être inadéquat en ce qu'il serait incapable d'expliquer le signe distinctif et fondamental de l'éthique, à savoir son caractère normatif. Je tente de montrer que la critique n'est pas pertinente, car le normatif n'a en réalité qu'une place dérivée en morale : l'éthique est d'abord une question de valeurs, entités dont il est tout à fait plausible de dire que nous les percevons. Pour justifier la place dérivée du normatif, je m'appuie sur les études de Max Scheler et sur l'examen de certains traits de la psychologie morale, qui tendent à montrer que le normatif n'est qu'un cas particulier de l'axiologique, mettant en évidence les caractères de force des valeurs, de risques de transgression et de contrainte.
\end{abstract}

ABSTRACT. - The perceptual model of moral knowledge has been the target of criticisms: it could be unable to explain the basic character of ethics, namely its normative character. I try to show that this criticism is misguided: in fact normativity is not central but lateral to ethics. What matters in ethics first and foremost are values, and it is quite appropriate to say we perceive values. To justify the lateral character of normativity, I rely on some analysis of Max Scheler and turn to some traits of moral psychology. It suggests that the normativity is a province of the axiological, characterized by strength of values, risks of transgression and constraint.

\section{Introduction}

Lorsqu'il examine le modèle perceptuel que John McDowell propose de la connaissance morale, Charles Larmore y décèle une erreur profonde : «Le défaut fondamental de cette conception, c'est qu'elle ne nous aide nullement à saisir le contenu normatif de la connaissance morale ${ }^{1}$. La connaissance morale est une connaissance normative, par quoi il faut comprendre que c'est " une connaissance de ce que l'on doit croire et faire $»^{2}$, par opposition à la connaissance factuelle, c'est-à-dire à la connaissance de ce qui est. Or la perception est une connaissance factuelle, donc elle ne peut convenir pour rendre compte du normatif.

L'argument est direct et plutôt efficace. Toutefois, il comporte certaines zones d'ombres, dont une va particulièrement nous intéresser : que faut-il entendre par « normatif ", par ce « doit » caractéristique de la connaissance morale et, à travers elle, de la morale elle-même ? Pour Larmore, cela a à voir avec la justifiabilité par des raisons et, dans cette optique, on comprend que

1. Larmore, Charles, "La connaissance morale ", dans Ogien, Ruwen, dir., Le réalisme moral, Paris, PF, 1999, p. 410.

2. Ibid, p. 408.

PHILOSOPHIQUES 28/1 — Printemps 2001, p. 69-86 


\section{Philosophiques / Printemps 2001}

son opposition à McDowell retient quelque chose de l'opposition entre le rationaliste et l'empiriste. Mais l'argument de Larmore n'est pas limité à ce contexte et il garde toute sa validité si, comme c'est souvent le cas, on lie le normatif à l'obligatoire : le modèle perceptuel paraît en effet tout aussi incapable d'expliquer le caractère obligatoire de la morale.

Et pourtant, nous pensons que, lorsqu'on y regarde de plus près, l'argument ne tient pas, parce que le caractère normatif de la morale n'est pas premier, mais dérivé, et que le modèle perceptuel n'a pas pour tâche de rendre compte de ce qui est ainsi dérivé en morale. Autrement dit : la place des normes, c'est-à-dire des énoncés moraux exprimant des devoirs ou des obligations, n'est pas centrale, mais latérale en éthique, ce qui place certes ces énoncés hors du champ du perceptuel, mais de façon totalement inoffensive pour ce modèle. C'est cela que nous allons tenter de justifier, en partant de l'examen de ce « doit » qui paraît aux yeux de beaucoup constitutif tant de la morale que du normatif.

\section{De quelques usages significatifs de « doit »}

«Doit» renvoie au devoir, notion morale fondamentale chez les déontologistes et bien au-delà. On appellera alors " normes morales » les énoncés exprimant un devoir moral, soit explicitement ( "Tu ne dois pas mentir ! ») soit implicitement («Tu ne mentiras pas !»). Toutefois, comme c'est bien connu, « doit » a encore d'autres usages. En voici quelques-uns, qui sont pertinents pour notre problème.

1) «Pour te rendre au Canada, tu dois prendre l'avion ». Ici, " dois » a la valeur d'un commandement conditionnel ("Si tu veux te rendre au Canada, ... »). Kant parlait d'impératif hypothétique, qu'il rattachait à la prudence et non à la moralité 3 .

2) Dans l'énoncé précédent, "dois » peut encore avoir la valeur d'une recommandation, car il serait aussi possible de prendre le bateau. $\mathrm{Si}$ " dois » est utilisé pourtant, c'est que les circonstances font juger que l'avion est le moyen de transport le plus adapté ou le plus raisonnable. Ce devoir se rattache donc au caractère rationnel qu'on attend de toute action, mais en un sens faible : si l'interlocuteur prend le bateau, on hésitera sans doute à dire qu'il s'est montré irrationnel.

3) "Tu dois prendre sa dame ", dit-on à un joueur d'échecs quelque peu inattentif. Recommandation sans doute, et de caractère hypothétique (si l'adversaire est mon enfant à qui j'apprends à jouer, je peux faire exprès d'épargner sa dame). Mais si quelqu'un a décidé de jouer, la « bonne pratique » lui demande de gagner ; s'il ne le fait

3. Kant, Emmanuel, Fondements de la métaphysique des moeurs, Paris, Vrin, 1980, p. 88. 
pas et qu'il n'a pas de bonne raison à cet effet, il se comporte de manière non seulement déraisonnable, mais encore irrationnelle.

Ces trois usages sont proches l'un de l'autre : chaque fois, le but étant fixé, les moyens s'imposent en quelque sorte, avec plus ou moins d'insistance, d'où l'emploi de " dois ». Chaque fois donc, quelque chose fait irruption avec une certaine force ; quand elle est au maximum, quand il n'y a pas d'alternative, on peut substituer " il est obligatoire de » à " tu dois ", quand elle est très faible, le conditionnel est de mise : " tu devrais ».

On peut souligner un autre point commun à ces trois usages : chaque fois, il s'agit d'une exigence de la raison, mais dans un domaine non moral. Cela signifie-t-il que le "doit » moral a la même grammaire, mais que c'est simplement son domaine d'application qui change ? Certains auteurs le pensent : "Pour faire le véritable bien de ton patient, tu dois obtenir son consentement » pourrait-on lire dans un Serment d'Hippocrate remis au goût du jour. Cette analyse toutefois est fortement contestée par les kantiens, on le sait, car la morale qui ressortit à la raison pratique n'est pas conditionnelle : "Si tu veux faire le véritable bien de ton patient... », elle est catégorique : «Tu dois obtenir le consentement de ton patient ! " La morale nous donne une tâche à accomplir, que nous le voulions ou non : la force du devoir moral est toujours maximale — c'est une obligation — et elle est d'une nature différente de la simple obligation de raison. On sait que, pour la caractériser, Kant parle d'impératif catégorique ; Max Scheler, qui ne se reconnaissait pas dans le déontologisme professé par le philosophe de Königsberg, préfère parler de "devoir comme tâche ${ }^{4}$, d'autres auteurs de " devoir-faire », d'autres encore, comme Richard Hare, de prescription.

Ainsi, ce qui caractérise les normes morales, c'est qu'elles nous donnent des tâches catégoriques à remplir, tâches qui sont au coeur de la morale et donc des normes qui les expriment ${ }^{5}$. Quelles sont ces tâches ? Pour le savoir il faut faire appel à ce que, justement, on nomme "éthique normative ", laquelle semble effectivement ressortir plus à l'exercice de la raison qu'à celui d'une perception : énoncer des normes dirigeant et justifiant nos conduites paraît être une activité de part en part rationnelle, analogue à la formulation et à l'application des lois dans les sciences.

Toutefois, quand on y regarde de plus près, on se rend compte que le devoir comme tâche n'épuise pas le champ de la morale. En effet, Scheler relève encore :

On peut parler de " devoir-être idéal » et lui opposer cet autre " devoir-être » qui est considéré en outre dans son rapport avec un vouloir possible destiné à

4. La notion de " tâche » est toutefois bien présente chez Kant, lorsqu'il dit de l'impératif catégorique qu'il " représente une action comme nécessaire pour elle-même" (Ibid., p. 85).

5. Nous ne dirons rien ici des normes sociales et institutionnelles, qui ont bien entendu des points communs avec les normes morales, mais dont le caractère catégorique est différent, si tant est qu'il existe. 


\section{2 · Philosophiques / Printemps 2001}

réaliser son contenu ( le devoir comme tâche »). Le premier cas correspond par exemple à la phrase : "Il ne doit pas y avoir d'injustice », le second à la phrase : "Tu ne dois pas commettre d'injustice $»{ }^{6}$

C'est que le "Tu dois ", qui s'adresse à une volonté, a un socle justificatif qui se trouve dans un "Il doit »; autrement dit, s'il y a tâche, c'est que quelque chose se présente comme n'existant pas encore et devant être réalisé, d'où l'épithète "idéal ». Il en résulte que la morale n'est pas tant caractérisée par la tâche que par l'idéal, thèse qui, chez Scheler, est à l'origine de nombreuses critiques dirigées contre Kant, car il estime que le philosophe de Königsberg a confondu les deux, au profit de la tâche ${ }^{7}$.

La distinction que propose Scheler ne se réduit toutefois pas à l'affirmation qu'il existe des normes impersonnelles, sources de devoirs s'adressant à tous les agents moraux : "Tu ne dois pas commettre d'injustice, car il ne doit pas y avoir d'injustice ", ce que nombre de philosophes lui concèderaient sans difficulté. Non, car d'une part « dois » et " doit » n'ont pas la même valeur, l'idéal n'étant pas une tâche, et d'autre part le fondement dernier de l'obligation n'est pas la norme, mais la valeur : «Le «devoir-être-à-titre-de-tâche» est donc quelque chose qui s'ajoute à un certain royaume axiologique, dans la mesure où les valeurs qui constituent ce royaume sont considérées dans la direction de leur réalisation par une tendance active possible ${ }^{8}$.

Si on laisse de côté le style quasi-platonicien de Scheler, on voit que la thèse de base qu'il défend, réduite à sa plus simple expression, est que les obligations se fondent sur les valeurs. Il lui arrive d'ailleurs de le dire sans ambages : "Tout ce qui possède une valeur positive doit être, et tout ce qui possède une valeur négative ne doit pas être. La corrélation ainsi définie n'est aucunement réciproque, mais strictement unilatérale. Toute obligation se fonde sur des valeurs, mais les valeurs ne se fondent jamais sur une obligation ${ }^{9}$. Bref, si je ne dois pas commettre d'injustice, c'est parce qu'il ne doit pas y avoir d'injustice ; et s'il en va ainsi, c'est parce qu'il n'est pas bon qu'il y ait de l'injustice ou, ce qui est équivalent, que l'injustice est un mal (elle possède une valeur négative). À l'origine, il y a les valeurs ; quand elles entrent en rapport avec la réalité (notre monde), elles aspirent à s'y réaliser (devoir-être idéal) ; d'où l'appel qu'elles nous adressent et l'exigence qu'elles nous imposent (devoir comme tâche).

6. Scheler, Max, Le formalisme en éthique et l'éthique matériale des valeurs, Paris, Gallimard, 1955, p. 202.

7. Cf. par exemple ibid., p. 202 : « Hegel a déjà montré à bon droit qu'une éthique qui, comme celle de Kant par exemple, se fonde sur le concept d'obligation, c'est-à-dire du devoir comme tâche, et qui voit là le phénomène originaire de l'éthique, ne peut jamais rendre compte de l'univers effectif des valeurs morales ».

8. Ibid., p. 203.

9. Ibid., p. 222. 
Pour notre propos, qui est de justifier la place latérale du devoir et de ménager la possibilité d'un modèle perceptuel de la morale, nous supprimerons un étage à cette conception et considérerons comme synonymes les énoncés «Il ne doit pas y avoir d'injustice » et "Il n'est pas bon qu'il y ait de l'injustice ». Peu importe ce que sont les valeurs en leur royaume : ici-bas, elle sont des idéaux (qui n'ont d'ailleurs rien d'inaccessible). Cela toutefois ne doit pas être compris comme la thèse soutenue par certains moralistes, que le devoir et la valeur, le normatif et l'axiologique sont équivalents : le " doit » qui habite la valeur n'est pas le même que celui qui habite l'obligation ; ainsi, nous ne considérerons pas l'énoncé "Il ne doit pas y avoir d'injustice" comme normatif, seul « Tu ne dois pas commettre d'injustice » ayant cette qualité ${ }^{10}$. Autrement dit, la morale est caractérisée par deux types de devoirs, l'idéal (" doit ») et la tâche ( " dois ») — le devoir-être et le devoir-faire, dit de nos jours avec bien d'autres Hector-Neri Castañeda ${ }^{11}$ — ; elle se réfère donc à deux types d'entités, la valeur (ou le bien) ${ }^{12}$ et l'obligation (ou le normatif), idéal et valeurs étant premiers, d'où le caractère latéral de la tâche et du normatif ${ }^{13}$. Cela permet d'entrevoir la réponse de principe que l'on peut donner à l'objection de Larmore contre le modèle perceptuel : si les valeurs sont au coeur de l'éthique, si le devoir moral est d'abord un « doit » et seulement ensuite un "dois ", alors ce modèle est recevable, car les valeurs sont justement des objets dont il paraît possible de dire qu'on les perçoit, ainsi que le souligne Stélios Virvidakis : « Le modèle des qualités secondes se prête mieux à l'explication du statut des propriétés qui font l'objet de jugements évaluatifs [...] plutôt qu'à l'explication du statut des propriétés intervenant dans des jugements prescriptifs ou déontiques $»^{14}$.

\section{La priorité de l'axiologique}

Ce qui précède reste assez général et appartient plus à l'ordre de l'exposition qu'à celui de la justification. Somme toute, pourquoi faudrait-il suivre Scheler

10. Il est clair que bien des auteurs utilisent l'expression "normatif » dans un sens plus large, incluant encore l'axiologique, le tout étant opposé au descriptif ; cet élargissement est parfois inoffensif, mais il ne manque toutefois pas de rendre le propos de ceux qui la commettent non pertinent pour notre problème.

11. Castañeda, Hector-Neri, Thinking and Doing, Dordrecht, Reidel, 1975, p. 46, qui précise, à propos du noème "Les femmes devraient enfanter sans douleur » qu'il " ne suggère en aucune manière qu'il y a quelque chose que les femmes doivent faire, mais seulement que le monde serait meilleur s'il n'y avait pas les douleurs de l'enfantement ». Cf. aussi p. 207-208.

12. Nous utiliserons souvent ces termes comme synonymes, bien que, stricto sensu, une valeur soit une propriété et un bien la chose qui possède cette propriété d'avoir une valeur. On nous pardonnera cette métonymie.

13. Certains seront peut-être tentés de dire que la morale (l'obligation, les normes) se fonde sur l'axiologie (les valeurs), mais cela ferait disparaître la question que nous examinons par un artifice définitionnel, ce qui ne saurait être recommandable.

14. Virvidakis, Stélios, La robustesse du bien, Nîmes, Chambon, 1996, p. 172. 


\section{Philosophiques / Printemps 2001}

plutôt que, disons, Kant ? En outre, si réellement le normatif est second par rapport à l'axiologique, en quel sens doit-on comprendre cette secondarité ? C'est ce que nous devons examiner maintenant, en tentant de justifier l'ordre de ces devoirs, c'est-à-dire l'antériorité des valeurs par rapport aux normes ${ }^{15}$.

\section{a) L'antériorité des valeurs}

Relevons avant toute chose que la position de Scheler n'est pas exotique : la priorité de l'axiologie est une thèse commune aux morales aristotéliciennes comme celle qu'il défend ${ }^{16}$ — et à l'utilitarisme : ce qui engendre normes et devoirs, c'est la considération de valeurs ou de l'une d'entre elles, par exemple l'utilité. Certes, d'autres doctrines aussi vénérables s'y opposent : le déontologisme, qui place les normes à la source des valeurs (ce n'est pas parce que la vie humaine est un bien qu'on doit la respecter ; mais elle est un bien parce qu'on doit la respecter ${ }^{17}$ ), et le contractualisme, d'où l'axiologie est même souvent absente, l'accord sur les normes occupant tout le champ éthique.

Qui a raison ? Les opposants à la priorité insistent souvent sur le fait qu'on ne voit pas comment on peut tirer « dois faire » de « est bon » — l'objection de Larmore à McDowell peut être interprétée ainsi - à moins bien sûr d'injecter déjà du normatif dans l'évaluatif. N'est-ce pas d'ailleurs ce dont Scheler s'est rendu coupable en introduisant sa notion " moyenne " de " devoir-être idéal », caractérisant déjà la valeur, avant toute tâche ou devoir faire ?

À cela, les partisans de la priorité répondent que, si l'on en reste aux usages ordinaires de "doit ", on pourra justifier à peu près n'importe quelle thèse, étant donné la plurivocité de cette expression, et donc qu'on peut gloser indéfiniment sur la valeur du " doit » que Scheler introduit pour caractériser les valeurs. Cela dit, continuent-ils, il faut rappeler que le « dois » des normes signifie un devoir fort, c'est-à-dire une obligation ; or, ainsi compris, il est assez raisonnable de soutenir que l'évaluatif n'implique pas toujours du normatif : de ce qu'une conduite est bonne, même ultima facie, il ne s'ensuit pas qu'elle soit obligatoire (les utilitaristes en disconviennent toutefois ${ }^{18}$ ). À quoi, bien entendu, les opposants rétorquent qu'il reste à expliquer pourquoi le passage a parfois lieu et, quand il a lieu, comment il peut avoir lieu. Bref,

15. Nous userons parfois d'un vocabulaire temporel : antériorité, précéder, etc. Mais, comme on s'en rend compte, nous ne lui donnons pas son sens propre, mais un sens conceptuel ou logique, ce qu'Aristote par exemple appelait l'antériorité de nature ; cf. Politique, I, 2, 1253 a 19, Paris, Vrin, 1970, p. 30 et Métaphysique, D, 11, 1019 a 2-5, Paris, Vrin, 1970, t. I, p. 282.

16. Les morales d'inspiration aristotélicienne ne se réduisent pas aux éthiques de la vertu ; l'eudémonisme en est aussi une variété, qui place à ses fondements un discernement approprié à une hiérarchie de valeurs; cf. notre ouvrage La valeur de la vie humaine et l'intégrité de la personne, Paris, PUF, 1995, ch. 1 et 2.

17. Comme l'exprime Charles Taylor: "Le bien est défini exclusivement par notre posture vis-à-vis de l'action obligatoire » ( « A most Peculiar Institution », dans Altham, James \& Harrison, Ross, dir., World, Mind, and Ethics, Cambridge, CUP, 1995, p. 136).

18. Singer, Peter, Questions d'éthique pratique, Paris, Bayard, 1997, p. 218-219. 
cette première réponse passe à côté de la véritable difficulté car, à défaut de la résoudre, rien n'empêche de nier la réalité du passage et donc la priorité de l'axiologique, soit que valeurs et normes soient indépendantes, soit que le passage ait bien lieu, mais dans le sens contraire.

Ainsi acculés, certains partisans préfèrent passer à l'offensive : concédant leur difficulté à rendre compte du passage (il n'est pas très clair de savoir comment il a lieu), ils font toutefois remarquer à l'adversaire que placer le normatif à l'origine ou le découpler des valeurs n'est pas plus satisfaisant, au contraire : si le normatif ne repose pas sur l'axiologique, quel est son fondement ? Est-il sui generis? Gare alors à l'inflation métaphysique; somme toute, les valeurs sont des propriétés bien plus "naturelles ». Est-ce la nature humaine ? Gare au paralogisme naturaliste ! Est-ce Dieu ? la raison transcendantale ? Mais peutêtre cherchons-nous trop loin; pourquoi ne pas faire appel aux décisions humaines, au consensus des théories contractualistes? C'est que vaut pour la morale ce qu'affirme David Gauthier du droit : "Dans un monde désenchanté, les prétentions normatives du droit ne peuvent être satisfaites que dans un cadre contractualiste ${ }^{19}$. Le problème n'est toutefois que repoussé d'un cran. Si l'on se met d'accord sur une norme de protection de la vie humaine, pourquoi adopte-t-on une telle norme et non son opposé ? Parce que nous avons peur de mourir ? Sans doute ${ }^{20}$. Mais cette peur, cette émotion, n'est-elle pas une réaction " perceptuelle » à ce mal qu'est la mort ? On peut, si l'on préfère, s'exprimer en termes d'intérêts : nous avons un intérêt à ne pas mourir ; mais ici encore, la question rebondit : n'est-ce pas parce que nous percevons la mort comme un mal ?

L'opposant à la priorité va-t-il rendre les armes ? Oui, s'il est inattentif. Car alors il ne remarquera pas que son adversaire utilise un argument qui ne touche pas vraiment sa position. En effet, ce qu'il montre, c'est que les normes morales renvoient à des valeurs, mais il n'établit pas par là que les normes morales reposent sur des valeurs morales. Or c'est cela qu'il devrait établir pour remporter la palme ; somme toute, tout moraliste pourra accepter sans trop de réticence que l'interdit du meurtre dépend de notre attachement à la vie, comme celui du vol de notre amour de nos possessions ; cela signifie simplement que notre morale est faite pour des êtres comme nous et non pour des ectoplasmes ou des divinités grecques. C'est là une thèse tout à fait inoffensive, et Kant lui-même reconnaît que les impératifs sont faits pour des personnes rationnelles comme nous, douées de dignité, mais capables de mentir ${ }^{21}$. C'est par contre tout autre chose que d'affirmer que l'interdit du meurtre

19. Gauthier, David, «Public Reason », Social Philosophy and Policy, 1, 1995, p. 40.

20. Rappelons qu'Ayer a développé son émotivisme pour résoudre la question des fondements et contrer ce qu'il appelle " la conception absolutiste de l'éthique »; cf. Langage, vérité et logique, Paris, Flammarion, 1956, ch. VI.

21. Et donc capables de mettre en danger leur dignité ; cf. Doctrine de la vertu, Paris, Vrin, 1985, p. 103 : "Le mensonge est abandon et pour ainsi dire négation de la dignité humaine ». 
repose sur la valeur (négative) de l'acte de tuer un innocent — il n'est donc pas vraiment correct de dire, comme nous l'avons fait, que pour un kantien, ce n'est pas parce que la vie humaine est un bien qu'on doit la respecter, mais elle est un bien parce qu'on doit la respecter ; il faut plutôt dire : le respect de la vie est un bien parce qu'il est commandé par l'impératif moral. Toutefois, il faut faire attention ici à une seconde chose : ce qui intéresse Kant, c'est la source de la valeur morale de nos actions et, à cet effet, il allègue la loi du devoir et la bonté de la volonté qui s'y conforme, alors que notre problème est différent ; il s'agit de savoir si le caractère normatif des impératifs est premier ou s'il se fonde sur une axiologie morale; bref, il ne s'agit pas de la source de notre valeur, mais de la source de la normativité. Cependant, sur ce plan aussi du rapport entre les normes morales et les valeurs morales, l'argument du partisan est simplement muet ${ }^{22}$.

L'examen de cet argument montre que la question est plutôt complexe : manifestement, il ne suffit pas d'étudier successivement ce que sont les normes et les valeurs morales pour voir s'établir une relation de priorité entre elles, quelle que soit d'ailleurs sa direction. Il faut donc, d'une manière ou d'une autre, élargir le débat. Une façon de le faire consiste à se hausser aux plans métaphysique et épistémologique, car la solution à la question de la priorité pourrait bien dépendre de thèses qui n'entrent en lice que là. Nous pensons notamment aux débats entre le réalisme et l'anti-réalisme, entre le cognitivisme et l'anti-cognitivisme. En effet, l'affirmation de la priorité des valeurs est souvent liée aux premiers termes de ces deux oppositions, celle de la priorité des normes aux seconds termes, même s'il existe des réalismes non perceptuels, tel celui soutenu par Larmore ${ }^{23}$. Nous nous proposons cependant d'emprunter une autre voie, moins fréquentée, celle de la psychologie morale. Il apparaîtra sans doute surprenant à beaucoup - c'est même peu dire — de vouloir résoudre un problème logico-conceptuel par ce biais. À cela nous répondrons trois choses : d'abord, nous ne prétendons pas que la psychologie va nécessairement trancher le débat, nous serions déjà heureux si elle pouvait l'éclairer, vu sa complexité. Ensuite, wait and see. Enfin, si tous les auteurs conviennent que la morale doit régler la conduite humaine, on l'a vu, et

22. On le voit, notre argumentation présuppose une distinction entre les valeurs morales et les valeurs non morales (qui, nonobstant l'épithète, ont une valeur pour la morale); nous en avons donné quelques éléments dans "Ombres et lumières de l'utilitarisme ", Revue de théologie et de philosophie, 4, 1998, p. 357-358, en nous inspirant de W. Frankena et de J.-Y. Goffi. Cette distinction est d'inspiration téléologique et relève d'une approche très différente de celle des déontologistes, qui ont tendance à la construire à partir de celle de l'altruisme et de l'intérêt personnel ; cf. Kekes, John, " On There Being some Limits to Morality », Social Philosophy and Policy, 2, 1992, p. 64 : «Les valeurs non morales ont à voir avec les bienfaits obtenus ou les maux évités d'abord par les agents pour eux-mêmes, tandis que les valeurs morales concernent les bienfaits et les maux que les agents causent à des tiers ». Cf. encore Brink, David, Moral Realism and the Foundations of Ethics, Cambridge, CUP, 1989, p. 213-217.

23. Larmore, "La connaissance morale ", p. 418, où il est question d'" une réalité normative ", à côté de "la réalité physique et psychologique ». 
qu'elle concerne la raison et la perception, qui sont elles aussi humaines, alors on ne sera pas étonné que l'examen d'attitudes humaines nous donne de précieux renseignements ; c'est plutôt le contraire qui serait surprenant.

\section{b) Le sentiment du devoir}

Dans le cadre de notre propos, nous placer du point de vue de la psychologie signifie que nous ne parlerons plus de normes, mais de sentiment de devoir (comme tâche) et de sentiment d'obligation. Dans quelles circonstances donc éprouvons-nous un sentiment de ce genre ? Laissons un peu voguer notre imagination.

- Le temps est splendide ; par ma fenêtre, j'aperçois les pentes enneigées des montagnes alentour. Je pense déjà avec délice à l'aprèsmidi de ski que je vais passer. Tout à coup, le téléphone sonne. Mon interlocuteur me rappelle que je lui dois un article sur les normes morales, que le délai imposé (que j’ai accepté) est déjà dépassé, qu'il a besoin de cet article sous trois jours. Je lui promets de m'y atteler immédiatement. Adieu montagnes, soleil et ski... C'est dur, mais je dois m'y mettre, c'est mon devoir.

- Je vais avec un ami à la librairie Maspero. Ayant choisi trois ouvrages, je m'apprête à me diriger vers la caisse, lorsque mon ami me dit : "Mets ces livres dans ta poche et sortons ; la librairie est tenue par des gauchistes qui n'osent pas intervenir contre la fauche ». Je le regarde avec réprobation, répliquant: "Mais cela ne se fait pas, c'est du vol, on doit payer ce qu'on se procure!»

Ces deux situations ont un point commun : il est fait appel au devoir pour contrer le risque d'une transgression morale, la sienne dans la première situation (si je suivais mon inclination, j'irais skier, et tant pis pour ce raseur !), celle d'un tiers dans la seconde. Autrement dit, on éprouve un sentiment de devoir en présence d'une menace pour la moralité et pour réaffirmer la force de cette moralité. Si cette menace n'existait pas, la première situation aurait une autre conclusion : non pas "C'est dur, mais je dois m'y mettre, c'est mon devoir ", mais quelque chose comme " Le ski sera pour une autre fois, pour l'instant j'ai mieux à faire ». Quant à la seconde situation, elle ne mériterait pas même une mention. Comment comprendre cela ? Pour Scheler, c'est le signe - la preuve ? - de la priorité de la valeur sur la norme :

Si je sais, par exemple, ce qu'il est bon que je fasse, peu me chaut ce que « je dois faire ". Pour que je doive, il faut d'abord que je sache ce qui est bon. Mais si je sais immédiatement et pleinement ce qui est bon, ce savoir affectif détermine également et de façon immédiate mon vouloir, sans que j’aie besoin de passer par l'entremise d'un « Je dois $»^{24}$.

24. Scheler, Le formalisme en éthique et l'éthique matériale des valeurs, p. 225. 


\section{8 · Philosophiques / Printemps 2001}

Le philosophe allemand va toutefois un peu vite en besogne : si « bon » est pris ici au sens de "moralement bon ", il ne va pas de soi que sa connaissance doive précéder celle de la norme, là est justement une partie du problème, que Scheler ne peut trancher ainsi que parce qu'il adopte la thèse en discussion, que la valeur précède la norme. Par contre, sur le plan de la motivation, le texte est en terrain plus ferme : sans aborder la question de savoir si une connaissance seule peut être motivante, fût-elle une perception-affective [fühlen] à la Scheler, il paraît juste de dire que si je sais ce qu'il est bien de faire, je n'ai pas besoin d'y adjoindre une connaissance de la norme correspondante pour le faire ; l'acte suit pour ainsi dire "naturellement ", sans contrainte, et même avec plaisir, si l'on en croit Aristote :

On n'est pas un véritable homme de bien quand on n'éprouve aucun plaisir dans la pratique des bonnes actions, pas plus que ne saurait être jamais appelé juste celui qui accomplit sans plaisir des actions justes, ou libéral celui qui n'éprouve aucun plaisir à faire des actes de libéralité, et ainsi de suite. S'il en est ainsi, c'est en elles-mêmes que les actions conformes à la vertu doivent être des plaisirs ${ }^{25}$.

Le sentiment de devoir est donc un sentiment de contrainte; on l'entend dire encore chez les philosophes rigoristes, qui précisent souvent que cette contrainte s'exerce contre nos penchants naturels, Kant bien sûr - qui précise toutefois qu'il n'en irait pas ainsi pour une volonté sainte ${ }^{26},-$ mais aussi Bergson : "Érigeons donc en maxime pratique que l'obéissance au devoir est une résistance à soi-même $»^{27}$. Pour eux, c'est là sinon le tout, du moins le coeur de la psychologie morale. Par contre, on le voit, il n'en va pas de même pour les aristotéliciens qui reconnaissent l'existence d'une autre attitude morale, que Scheler nomme " amour " et que, plus prosaïquement nous appellerons « attrait du bien »-car si le devoir pousse, le bien attire ${ }^{28}$. Or, et c'est là un point important, ces mêmes auteurs placent cet attrait audessus de l'attitude d'obligation: "On n'est pas un véritable homme de bien » dit Aristote, et Scheler : " L'attitude de la «belle âme» n'est pas égale à celle de l'homme de devoir, elle est supérieure en valeur ${ }^{29}$; et pour bien marquer la différence d'avec le rigorisme kantien, il ajoute :

C'est ce que ses principes interdisent à Kant d'accorder, puisque pour lui le mot " bon » n'assume une signification qu'à partir du concept d'obligation idéale, sinon, comme l'enseignent beaucoup de passages, à partir de l'idée de conformité au devoir. Aussi le bon accompli «par pure inclination » représente-t-il pour lui une contradictio in adjecto.

25. Aristote, Éthique à Nicomaque, 1099 a 17-21.

26. Kant, Fondements de la métaphysique des moeurs, p. 85.

27. Bergson, Henri, Les deux sources de la morale et de la religion, éd. du Centenaire, Paris, PUF, 1959, p. 991.

28. Les scolastiques parlaient d'un appétit, y compris pour désigner la volonté : « Appetitus intellectivus, scilicet voluntas " (Thomas d'Aquin, Somme théologique, Ia, q. 82, a. 2, ad 3).

29. Scheler, Le formalisme en éthique et l'éthique matériale des valeurs, p. 242. 
La supériorité de valeur alléguée ici n'est pas ce qui nous intéresse - on l'a dit, ce n'est pas la question de la source de notre valeur qui nous occupe — sinon dans la mesure où elle est l'expression, chez Scheler, de l'antériorité des valeurs sur les normes, et le signe chez Aristote de l'exclusion (implicite) des normes du domaine de la morale. Un tel ostracisme ne serait, actuellement, plus recevable, mais il est clair que, dans la mesure où le sentiment du devoir n'est qu'une sorte de pis-aller, on doit conclure que le devoir ne saurait être au centre de la morale : il est bien, en ce sens, latéral. Une morale sans obligation est à la limite concevable - c'était probablement la morale d'avant la chute et peut-être celle d'Aristote - une morale sans valeurs ne l'est en aucun cas.

Cette conclusion est-elle vraiment assurée ? On peut en douter pour deux raisons :

1) Le sens du mot "devoir " que nous venons d'examiner, c'est-àdire tel qu'il apparaît dans l'expression "sentiment du devoir » n'est peut-être pas le sens le plus approprié lorsqu'il est question de norme, ou du moins, il existe peut-être encore d'autres acceptions pertinentes dont la grammaire est différente.

2) Ce qui précède a été introduit comme un morceau de psychologie morale ; or, en conclusion, nous avons quitté ce cadre pour parler de la morale elle-même : le devoir ne saurait être au centre de la morale, avons-nous affirmé. N'est-ce pas un saut injustifiable ? Comment en effet tirer de la psychologie des thèses portant sur la structure logico-conceptuelle de la morale ?

La première objection va nous obliger à poursuivre l'examen, ce que nous allons faire sous peu. Auparavant toutefois, il nous faut nous occuper de la seconde. La psychologie nous dit effectivement quelque chose sur l'épistémologie et, à travers elle, sur la morale elle-même : si le devoir n'apparaît que dans des contextes de contrainte liée à une transgression menaçante et qu'il n'est pas légitime de réduire le domaine de la morale à ce type de situation, alors notre connaissance morale ne peut être limitée à ce qui est obligatoire, si bien qu'il existe nécessairement d'autres objets moraux. Mais peut-être que, sans y être limitée, la notion de devoir est-elle le pivot de la morale, nonobstant les leçons de la psychologie ? On ne peut l'exclure c'est justement le problème qui nous occupe - mais nous pensons justement que ce petit morceau de psychologie morale tend à montrer qu'on peut voir les choses différemment, à moins bien sûr que nous soyons ici en présence d'une illusion systématique, ce qui, lorsqu'il est question de psychologie, ne peut jamais être tout à fait exclu : le sentiment du devoir est l'émotion appropriée lorsque le bien moral n'attire pas comme il le devrait, et s'il en va ainsi, c'est qu'il est probable que l'attitude de l'homme de devoir soit l'attitude convenable dans des situations telles que la faiblesse de la volonté. C'est pourquoi une morale sans devoir est concevable, et donc que la notion de valeur est plus fondamentale que celle d'obligation. 


\section{Philosophiques / Printemps 2001}

\section{c) Le devoir corrélatif au droit}

Existe-t-il d'autres usages du terme «devoir » (comme tâche) que nous aurions laissé de côté ? C'est manifestement le cas. Lorsqu'on dit qu'un devoir est corrélatif à un droit, on ne fait pas référence à une transgression, même si l'aspect de contrainte reste présent, allié à la force de la considération morale. Développons un peu.

Admettons d'abord que la notion de droit moral a un sens, que ce n'est pas, ainsi que le pensait Bentham, "une absurdité sur pilotis ». Comme on sait, on divise de manière classique les droits en deux catégories, les droitslibertés et les droits-titres, auxquels correspondent respectivement des devoirs de non interférence et des devoirs d'assistance. Dans le cas des seconds, l'aspect de contrainte est évident, mais il n'est pas absent des premiers : il faut se garder d'intervenir. Par exemple, dans la doctrine du consentement libre et éclairé du patient, le médecin a d'abord un devoir d'assistance : donner au patient les informations nécessaires à la prise de décision, mais en fin de compte, il a le devoir de respecter le choix du malade, même si c'est un refus qu'il désapprouve. Cette désapprobation n'indique toutefois pas forcément qu'il soit tenté par la transgression et désire intervenir intempestivement.

La menace d'une transgression n'est donc pas caractéristique de cette forme de devoirs; toutefois il n'en va plus ainsi si l'on en regarde l'origine. On le sait, l'affirmation des droits, et particulièrement des droits-libertés, a accompagné la lutte contre l'absolutisme, contre lequel des objections morales fortes étaient dirigées ; dans les termes de Locke, "il s'agissait d'établir le droit égal que chacun a à sa liberté, et qui fait que personne n'est sujet à la volonté ou à l'autorité d'un autre homme ${ }^{30}$. Autrement dit, il s'agissait de dénoncer des transgressions morales - essentiellement ici des abus de pouvoir - et d'établir, corrélativement, un devoir de respect par rapport à certaines valeurs. Ainsi en va-t-il encore pour le consentement libre et éclairé : c'est en réaction au paternalisme médical que la doctrine a été formulée.

Le cas des devoirs d'assistance est un peu différent. En outre, il n'est pas facile à formuler en raison de la tournure qu'a prise la rhétorique morale de nos jours; en effet, on use et abuse actuellement du langage des droits, toute revendication morale s'en revêt ; le petit enfant déjà proclame : "J'ai tous mes droits ! » Reste à savoir lesquels... À l'opposé, certains philosophes, comme les libertariens et les anarcho-capitalistes, nient l'existence des droitstitres. Toutefois, dans l'esprit de ceux qui y ont recours, on saisit l'idée suivante : si chacun doit pouvoir mener une vie de son choix, il est nécessaire qu'il dispose de certains biens ; c'est particulièrement la position de tous ceux qui s'inspirent de l'approche de Rawls, dont van Parijs dit notamment ceci : "Sur quoi peut reposer la liberté réelle sinon sur ces avantages socioéconomiques dont nous avons besoin pour réaliser nos projets de vie, quelle

30. Locke, John, Traité du gouvernement civil, Paris, GF, 1984, p. 183. 
que soit la nature exacte de ceux-ci ? ${ }^{31}$. D'où, par exemple, l'idée du droit à un revenu minimum ou à des soins médicaux de base. Le devoir corrélatif à ces droits ne s'adresse en général qu'indirectement aux individus, ce sont des institutions sociales, voire la communauté politique qui doivent s'en charger ; c'est pourquoi, si l'idée de transgression n'en est pas forcément absente, c'est plutôt celle de négligence qui les accompagne : l'État, entendon dire, ne s'occupe pas assez des citoyens, il les laisse (trop) livrés à euxmêmes. Mais cette négligence n'est dénoncée que lorsqu'elle porte sur des biens qui ont une certaine importance pour tout un chacun : le revenu, le travail, la subsistance, le logement, les soins, etc. Sinon, on n'invoque ni droits ni devoirs. On rejoint donc ici l'autre aspect de la notion de devoir, celui de la force d'une considération morale.

Cette rapide phénoménologie du devoir comme corrélatif à un droit devrait suffire pour l'examen de la question de son rapport aux valeurs. Nous avons parlé du respect de certaines valeurs, nous avons mentionné la force de certaines considérations morales; on interprétera facilement ces dernières comme des intérêts, dont on a indiqué le lien aux valeurs (on a intérêt à quelque chose, qui est toujours un bien) ou même tout simplement comme des valeurs. Cela indique-t-il que les devoirs sont seconds par rapport aux valeurs ? Sans doute et, dans une certaine lecture, on a immédiatement la thèse que nous soutenons. Griffin, par exemple, en parlant « des valeurs que les droits protègent", mentionne : "l'autonomie, la liberté et l'égal respect »32. Or, l'égal respect est bien une valeur morale. Toutefois, à y regarder de plus près, on en vient à se demander si c'est bien cette valeur que le droit protège, ou s'il ne faut pas plutôt dire que cette valeur est exprimée par la norme. Et alors, on se rend compte qu'il existe une autre lecture de la priorité des valeurs qui, à nouveau, est totalement inoffensive : le devoir (comme tâche) de protéger la vie humaine signale l'importance de la valeur de la vie humaine, mais la vie humaine n'est pas une valeur morale. C'est le respect de la vie qui en est une. Et il en va exactement de même pour l'autonomie, la liberté et l'égalité ! Chaque fois, le respect est exprimé par une norme ; est-il constitué par elle ? Il semble que nous soyons ramenés à notre point de départ.

Ne soyons toutefois pas trop pessimistes, car ici comme avant, nous pouvons observer un phénomène constant : toute valeur morale n'est pas associée à une norme, l'évaluatif moral déborde encore le prescriptif. Le cas de l'amitié est paradigmatique sur ce plan depuis au moins Aristote. Dans le cas du sentiment de devoir, nous avions vu que ce qui faisait que l'on passait de l'axiologique au normatif, c'était le risque de transgression et la contrainte; qu'en est-il ici ? C'est, on l'a déjà indiqué, principalement la force : certaines considérations morales ont tant de force (dans l'absolu ou

31. van Parijs, Philippe, Qu'est-ce qu'une société juste ?, Paris, Seuil, 1991, p. 210.

32. Griffin, James, Well-Being, its Meaning, Measurement and Moral Importance, Oxford, Clarendon, 1986, p. 235. 


\section{2 - Philosophiques / Printemps 2001}

dans telle situation donnée) qu'on les exprime dans le couple droit-devoir. Bref, le devoir est ici le signe de la force de la valeur, tellement que la norme apparaît bien comme seconde.

Si on n'y fait pas toujours attention, c'est que la rhétorique morale contemporaine tend à l'estomper et à faire croire que toute considération morale doit faire l'objet d'une formulation en termes de droits (et de devoirs, concède-t-on du bout des lèvres). Il est possible, si l'on suit cette voie pendant un certain temps, que la grammaire morale s'en trouve modifiée. Mais selon nous ce serait néfaste, car la notion de droit associant souvent les deux idées d'importance morale et de menace de transgression, la vie morale se verrait vécue sur un mode de plus en plus unidimensionnel, celui d'une revendication fondée sur un antagonisme.

C'est là une leçon qui concerne la vie éthique ; on peut encore en tirer une seconde, qui a trait à la logique de la morale : nous avons parlé d'antériorité ou de priorité de l'axiologique, mais sans préciser beaucoup. Nous voyons maintenant qu'il ne s'agit pas de comprendre cela au sens où le normatif pourrait être tiré de l'axiologique par une inférence quelconque. Le normatif apparaît plutôt comme une province de l'évaluatif, caractérisée justement par la force, la contrainte et la menace de transgression. Mais avant d'aller plus loin sur ce terrain, nous devons encore examiner si nous n'avons rien laissé d'essentiel de côté.

\section{d) De quelques autres acceptions}

Les deux usages du terme "devoir » que nous avons examinés nous paraissent les plus importants et les plus constants. Mais il y en a d'autres, de plus régionaux. Examinons-en rapidement quelques-uns, afin de voir s'ils peuvent encore nous apprendre quelque chose, confirmer ou infirmer nos conclusions provisoires :

1. Le devoir ou la norme signalent l'objectivité, par opposition à la subjectivité des valeurs. Dans cette optique, Emmanuel Picavet relève : «Subsiste, en effet, la possibilité que le «bien» et le «meilleur» ne soient pas donnés simplement subjectivement, mais soient au contraire (ou peut-être par surcroît) appréhendés de manière objective. C'est sous les espèces du devoir que la tradition philosophique conserve la trace de cette approche de l'action ${ }^{33}$. C'est là une acception qu'adoptent volontiers les théoriciens du choix rationnel et certains déontologistes, comme Habermas, lorsqu'il affirme, interprétant l'objectivité sous les auspices de l'universalisme et la subjectivité sous celles du particularisme :

Les questions morales, qui peuvent, fondamentalement, être décidées rationnellement sous l'aspect de la justice, ou de l'universabilisation des intérêts, sont désormais distinguées des questions évaluatives qui, sous leur

33. Picavet, Emmanuel, Choix rationnel et vie publique, Paris, PUF, 1996, p. 106. 
aspect le plus général, se présentent comme des questions concernant la vie bonne (ou de la réalisation de soi), et qui ne sont accessibles à une explication rationnelle qu'à l'intérieur de l'horizon non problématique d'une forme de vie historiquement concrète ${ }^{34}$.

On voit facilement que cette conception du devoir et de la valeur exige, pour être justifiée, un appareil théorique lourd, car avec un autre appareil il serait également plausible de soutenir l'objectivité des valeurs ou la régionalité des normes ${ }^{35}$. Or, autant que faire se peut, nous désirons n'examiner que les propriétés des valeurs et des normes qui font l'objet d'un accord large, à défaut d'être unanime.

2. «L'impératif ou le prescriptif portent exclusivement sur des actions de type volontaire non encore accomplies. La portée de l'appréciatif est beaucoup plus large, puisqu'il couvre également les actions volontaires accomplies, les actions involontaires, les états mentaux, les dispositions, les objets, etc. ${ }^{36}$. Ici, il s'agit d'une question de signification concernant, comme le texte le dit, la portée des expressions : les domaines respectifs du normatif et de l'évaluatif (appelé par Ogien « appréciatif») sont différents. Plus précisément, le domaine du second comprend celui du premier. Or, c'est exactement ce que nous avons affirmé, si ce n'est que nous nous sommes limités à cette partie de l'appréciatif que constitue l'axiologique moral, et en ce sens les remarques faites ici ne sont en aucun cas une objection à la thèse que nous défendons : le normatif est un cas particulier d'axiologique. Simplement, alors que nous avons insisté sur la menace de la transgression et sur la force de la valeur, le texte cité identifie le type de domaine moral que ces propriétés caractérisent : les actions volontaires futures. Si la précision est bienvenue, il faut tout de même relever qu'elle ne suffit pas comme critère de démarcation car, Ogien en convient d'ailleurs explicitement, les actions volontaires futures sont aussi parfois l'objet d'évaluations non normatives pensons, encore une fois, aux actions qui sont le fruit de l'amitié.

3. On oppose souvent les devoirs (et donc les normes) de justice aux évaluations du bien. Et il faut reconnaître que la justice est éminemment la source d'énoncés normatifs et de revendications en termes de droits et de devoirs. Mais cela amène-t-il quelque chose de nouveau pour notre propos ? Heureusement, pour y répondre, nous n'aurons pas besoin de proposer quelque chose comme une théorie de la justice, car il existe une réponse courte. Comme le dit Habermas, "la distinction déontologique entre le juste et le

34. Habermas, Jürgen, De l'éthique de la discussion, Paris, Cerf, 1992, p. 39-40.

35. Cf. par exemple cette remarque de Robert Attfield, faite sans la moindre hésitation : "Les choses sont comprises ici comme ayant de la valeur lorsqu'il existe des raisons interpersonnelles pour les promouvoir ou les préserver » ( "The Global Distributive of Health Care Resources ", dans Lesser, Harry, dir., Ageing, Autonomy and Resources, Aldershot, Ashgate, 1999, p. 136).

36. Ogien, Ruwen, "Normes et valeurs ", dans Canto-Sperber, Monique, dir., Dictionnaire d'éthique et de philosophie morale, Paris, PUF, 1996, p. 1054. 


\section{Philosophiques / Printemps 2001}

bien correspond à celle opérée entre les jugements normatifs et les jugements évaluatifs ${ }^{37}$. Bref, quelque opposé qu'il soit au bien, le normatif se fonde aussi sur une valeur morale : le juste, dont la force sociale n'échappera à personne. Il suffit donc d'en prendre acte ${ }^{38}$.

4. Jusqu'ici, nous avons parlé des devoirs qu'on pourrait dire " naturels ». Mais il en existe encore une autre sorte, ceux qui sont acquis; beaucoup ressortissent moins à la morale qu'à la déontologie, mais la ligne qui sépare ces deux domaines n'est pas très nette, notamment si nous pensons aux différents rôles sociaux que nous remplissons, dans la vie socio-politique, la vie professionnelle et la vie familiale. Toutefois, il existe des cas clairs, par exemple les contrats. Ils créent des devoirs qui signalent quelque chose que nous n'avons pas encore considéré : l'engagement réciproque de personnes, soit directement les unes envers les autres, soit indirectement, par le biais d'institutions (et ici, nous sommes très proches du juridique). Or, ces devoirs priment sur certaines valeurs, comme le souligne Frances Kamm : si six personnes sont en danger de mort, que je ne peux les sauver toutes et que j'ai un devoir contractuel de sauver les cinq premières, je dois le faire, même si la sixième est un ami très proche ${ }^{39}$. Cela signale-t-il une primauté du normatif ? Non, car la primauté que nous observons ici n'a rien à voir avec notre question : il s'agit d'un conflit moral qui peut tout aussi bien être interprété comme un conflit de valeurs que comme un conflit de normes. La primauté alléguée ici est d'ailleurs elle-même discutable ; pensons au cas où la sixième personne serait mon enfant. Pour notre propos, ce cas ne signale donc rien de nouveau, il est redevable de la même analyse que celle des devoirs corrélatifs à des droits, si ce n'est qu'il s'agit de devoirs choisis qui, souvent, ne sont importants que subjectivement, pour les contractants eux-mêmes.

\section{Conclusion}

Sans prétendre avoir été exhaustifs, il nous semble avoir examiné suffisamment d'aspects de la question pour pouvoir affermir notre conclusion. Mais résumons d'abord notre argumentation. Larmore contestait à la conception perceptuelle de la morale la capacité de rendre compte de l'aspect normatif de la morale. Nous avons tenté de montrer que ce n'était pas le cas en distinguant à la suite de Scheler deux sens du terme "doit » en morale, le devoir-être idéal et le devoir comme tâche, le premier caractérisant les valeurs, le second les normes comprises comme impératifs. Nous avons

37. Habermas, De l'éthique de la discussion, p. 151.

38. Soulignons que le problème qui nous occupe dans cet article ne peut être réduit à celui de la priorité du bien par rapport au juste, caractéristique de l'opposition entre le libéralisme et le perfectionnisme. Par contre, nos propos peuvent mettre en lumière certains arrière-plans de cette opposition.

39. Kamm, Frances, Morality, Mortality, Oxford, OUP, 1993, vol. 1, p. 77. 
ensuite soutenu que ce qui est au coeur de la morale, c'est le devoir-être idéal, c'est-à-dire l'axiologique, et non le normatif. Ce dernier s'est alors révélé comme une spécification de l'axiologique dans deux types de situations qui, parfois, peuvent se conjoindre : la menace de transgression et la force particulière d'une valeur, d'où le caractère de contrainte du prescriptif. Ainsi, le normatif est bien second - comme le dit Kevin Mulligan : « Les valeurs sont plus fondamentales que les normes $»^{40}$ - mais il ne s'agit pas de le «tirer» de l'axiologique, ni de l'en déduire, encore moins de l'y réduire ; on pourrait dire du normatif qu'il n'est que l'axiologique considéré sous certains points de vue particuliers, paraphrasant ce que disait Cabanis du mental - qu'il appelait « moral » - au début du XIX ${ }^{\mathrm{e}}$ siècle : " Le moral n'est que le physique, considéré sous certains points de vue particuliers $»^{41}$. Il faut toutefois se méfier de ce genre de comparaisons qui peuvent laisser croire que l'axiologique et le normatif sont en définitive identiques. Il est plus éclairant de se placer sur le plan de la syntaxe et d'affirmer en nous inspirant de Castañeda que les valeurs sont des propriétés des actions, alors que l'obligation est un opérateur dont le langage use pour souligner que la valeur de l'acte est une forte raison d'agir, souvent dans un contexte de réticence à agir ${ }^{42}$ — et ici, on retrouve l'intuition de Larmore.

La valeur et son type particulier de normativité, si l'on peut s'exprimer ainsi pour désigner l'idéal, étant remise au centre de la morale, le modèle perceptuel lui étant par ailleurs adapté, la difficulté soulignée par Larmore est levée $e^{43}$. Toutefois ce modèle rencontre aussi des difficultés, dont l'une est proche de celle que soulevait Larmore ; comme le souligne Virvidakis lors de son examen des difficultés de la position de MacDowell, comparant les valeurs aux qualités secondes : "Dans le cas des valeurs on fait explicitement référence à une dimension normative, qui est absente dans des qualités secondes comme la couleur $»^{44}$. Cela reste vrai : le devoir-être idéal ressortit au « devoir-être » et non à l'" être "; il faut donc formuler un modèle perceptuel qui s'en accommode. La solution pourrait bien se trouver du côté d'une doctrine faisant jouer un rôle central aux émotions et aux sentiments

40. Mulligan, Kevin, "From Appropriate Emotions to Values ", The Monist, 1, 1998, p. 166. Nous ne sommes toutefois pas sûr que cet auteur serait d'accord avec notre interprétation de l'expression "plus fondamental ».

41. Cabanis, Pierre-Jean-George, Rapports du physique et du moral de l'homme, I, 3, Genève, Slatkine reprints, 1980, p. 78.

42. Castañeda, Thinking and Doing, pp. 179 et 337. L'auteur aborde toutefois son sujet dans un tout autre cadre que le nôtre, celui du prescriptivisme.

43. Cf. ce que dit Ogien dans son introduction au Réalisme moral, p. 55 : «Le réaliste moral peut se permettre de reconstruire l'idée de vérité en éthique à partir d'énoncés évaluatifs tels que "L'épicier est malhonnête" dont la forme déclarative n'interdit pas, à première vue, qu'on puisse dire d'eux qu'ils sont "vrais" ou "faux", ce qui, ajoute-t-il, serait plus difficile à "partir d'énoncés prescriptifs de forme impérative tels que "Épicier, ne sois pas malhonnête !” ".

44. Virvidakis, La robustesse du bien, p. 167. 


\section{6 · Philosophiques / Printemps 2001}

comme « organes » de perception ${ }^{45}$. Placer l'axiologique au centre du moral a bien d'autres conséquences encore, comme celle de montrer que le sentiment d'aliénation que nous éprouvons vis-à-vis de la morale, dont parle Griffin, venant $\mathrm{du}$ "fait » que : "Nous ressentons la moralité comme quelque chose qui est extérieur au fonctionnement de notre propre nature, comme quelque chose d'imposé de l'extérieur » ${ }^{46}$ n'est pas un sentiment approprié vis-à-vis de la morale comme telle. Cela a encore des conséquences pour la question de savoir quelles sont les émotions et sentiment moraux de base ; mais sur tout cela, nous nous contenterons des quelques remarques faites en cours de route.

45. Cf. notamment, outre le travail de pionnier de Scheler, Christine Tappolet, «Une épistémologie pour le réalisme axiologique », dans Ogien, Ruwen, Le réalisme moral et, plus récemment, Émotions et valeurs, Paris, PUF, 2000.

46. Griffin, Well-Being, its Meaning, Measurement and Moral Importance, p. 127. 